\title{
A microprocessor-based psychopathology laboratory: III. Hardware
}

\author{
LAWRENCE G. SPACE and RAND S. HUNTZINGER \\ School of Medicine and Dentistry, Department of Psychiatry, University of Rochester, \\ Rochester, New York 14642
}

\begin{abstract}
The hardware configuration of a psychopathology research and clinical laboratory is described. Design prerequisites that guided hardware selection include: flexibility in experimental control, ease of data handling, system mobility, operational simplicity, and a method of providing clinical feedback. The microcomputer, mass storage, digital input/output, timers, and the auditory and visual modes of interacting with subjects/patients are described. Component costs are illustrated.
\end{abstract}

The psychopathology laboratory at the University of Rochester School of Medicine and Dentistry was designed to implement a wide variety of experimental procedures. The rationale for such a laboratory, the software created, and the types of research that have guided design considerations are discussed by Cromwell and Spaulding (1979), Huntzinger and Space (1979), and Spaulding and Space (1979).

This paper looks more closely at the design goals and how these goals were implemented through the selection of computer hardware and peripherals. In broad terms, the design prerequisites that guided system development were: flexibility in experimental control, ease of data handling, system mobility, operational simplicity, and clinical feedback with minimal turnaround time.

\section{DESIGN PREREQUISITES}

\section{Flexibility in Experimental Control}

The Psychopathology Laboratory was created with the potential for research and clinical applications that cover a wide range of human functioning. Both cognitive and physiological aspects of human functioning are of interest to our research group. For general research instructions, psychological testing applications, tachistoscopic work, and biofeedback research, a method of conveying auditory and visual information was considered necessary. In addition, for the system to accept responses from a subject, a method of entering verbal statements and buttonpresses was desirable. The ability to collect physiological data such as EEG, EKG, and EMG is currently being developed and yet to be fully implemented. To handle these kinds of data, the computer system must be able to handle both analogue and digital signals as input and output. To respond to changing research needs, it was desirable to have an expandable system capable of accepting a wide variety of peripheral

Acknowledgment is offered to NIMH Grant MH-14650 and New York State Health Research Council Grant HRC 846. equipment. With these features the laboratory can grow and be able to control a wide range of experimental situations.

\section{Ease of Data Handling}

Human transformation and handling of raw data is a time-consuming, error-prone, and expensive aspect of research endeavors. The use of on-line computers to collect, transform, and store data substantially reduces the error rate, toil, and cost of data handling. The laboratory was designed to minimize operator and clerk contact with a subject and his/her data and to maximize the time spent on research design and development. To meet these general goals, a method of mass data storage, a communication link to large mainframe computers, a method of direct CRT display of physiological signals, a simple method of backup storage for computer programs and data, and on-line interactive capability were acquired. The backup system allows recovery from unforseen operator or system errors.

\section{Simplicity of Operation}

Of prime consideration in the laboratory design was ease of operation for the end user, who may be a person of minimal training in the use of computers or even experimental procedures. It was also important to provide tools for program development for the sophisticated user. Ease of operation is enhanced by quick start-up time, a simple programming language, a language with file management capability, hard copy, software editing, and ease of compilation and testing of software.

From the time a subject is "hooked up" for an experiment until its conclusion, the computer controls the course of events as much as possible. This includes oral and written instructions, recognition and processing of subject errors, and automatic collection of data.

\section{System Mobility}

At some point in the future there may arise a need to go to the subjects, that is, to put our laboratory on 
wheels and visit various clinical populations at some distance from our present location. A stand-alone system not dependent on a larger computer is one way to achieve this kind of mobility. Alternately, by choosing a computer that is instruction set compatible with at least one of the mass produced commercially available low-cost microcomputers, it is possible to set up satellite systems with minimum software changes. The recent development of nearly machine-independent languages, such as PASCAL, permits efficient transfer of programs from computer to computer, whether or not the processor is similar. The satellite systems, if acquired, will be dedicated to specific tasks, while the larger microcomputer system will provide a central data base and a means of program development.

One further advantage to a stand-alone installation is its relative independence from the vagaries of university computer down times, a not uncommon experience when a large number of users are served.

\section{Clinical Feedback}

Since providing clinically relevant feedback to referring agents is an important projected use of the system, a method of generating written reports was needed. A local hard-copy device and linkage with a large computer for sophisticated statistical analyses, such as factor analysis, was desired. The high-speed line printers available on a large computer also facilitate this process.

\section{Additional Considerations}

In addition to the five areas outlined above, local availability, local servicing, and acceptable delivery times were important factors guiding the choice of hardware. When the laboratory was conceived, an equipment order was placed; after waiting 12 months for a system that was promised within 90 days, the order was cancelled.
With these various considerations in mind, the basic system was purchased and is operational. To elaborate on the details of the hardware configuration, the system is described in four segments: microcomputer, mass storage, digital input/output lines and timers, and audio/visual/subject response. Figure 1 shows a block diagram of the entire system.

\section{HARDWARE CONFIGURATION}

\section{The Microcomputer}

The microcomputer is an IMSAI mainframe with a 19-slot Wunderbus and a Technical Design Laboratory Z80-based microprocessor board. The IMSAI mainframe and its $\mathrm{S}-100$ bus provides considerable expandability as well as being compatible with inexpensive peripheral interfaces supported by many vendors. Although many different bus structures are available in the microcomputer industry, the $S-100$ bus still appears to be supported by more peripheral manufacturers than any other bus. The Z80-based microprocessor has, as a subset, the 8080 instruction set, but provides additional instructions that, among other things, ease the handling of interrupts and block data transfers. This particular microprocessor is also used by several widely available low-cost computer systems that may be used for satellite systems.

The 64K of 250-nsec static RAM on the system is composed of four 16K Problem Solver memory boards. This amount of memory will handle large programs, process several multiplexed physiological signals, and enable the use of the PASCAL language with ease. By confining the memory to four boards, ample expansion slots remain. The $250-n s e c$ speed allows operation of the $\mathrm{Z} 80$ at $4 \mathrm{MHz}$ if desired, although it is currently operating at $2 \mathrm{MHz}$.

The basic operating system is $\mathrm{CP} / \mathrm{M}$ by Digital Re-

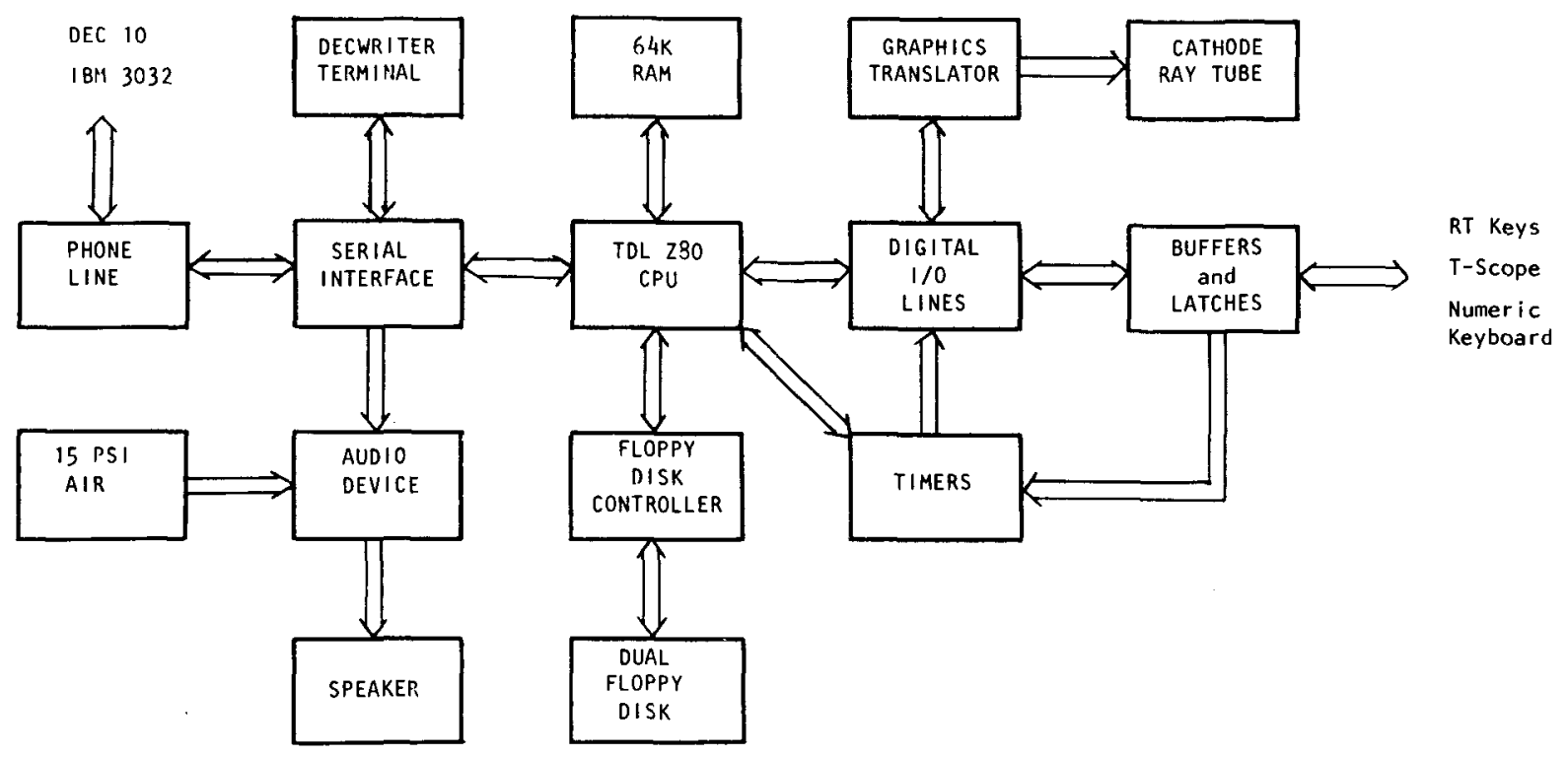

Figure 1. Laboratory microcomputer system block diagram. 
search. The system supports CBASIC, an easy-to-use high-level programming language for the nonsophisticated computer user, as well as a text editor useful for program development. In addition, FORTRAN IV and a relocating/linking assembler, both supported by TDL, were purchased. FORTRAN provides data and file management, while assembler eases the development of efficient system subroutines and programs that can be linked to FORTRAN. Shortly, PASCAL will be on the system; it appears to have considerable promise as a nearly machine-independent (Bowles \& Hollan, 1978) high-level programming language. We anticipate that it will slowly take over our operation as the language of choice.

\section{Mass Storage}

Two forms of mass storage are available on the laboratory system, local and remote. The local mass storage is provided by a double-density dual floppy disk system with an FDC3 control board by Digital Systems, Inc. The remote storage is provided by the disks and magnetic tapes available on either an IBM 3032 or a DEC-10 computer housed elsewhere on the University of Rochester campus. The floppy disk system was chosen because of its random access capability, ease of changing from one set of experiments or data bases to another, quick load time for programs, and, with additional diskettes, an unlimited amount of storage. The double-density operation provides for $512 \mathrm{~K}$ bytes per diskette, providing a megabyte of storage.

The dual-drive floppy system enables copying from one drive to the other to create backup diskettes for both progams and data. This has already proved in valuable because of an intermittent noise problem that resulted in numerous errors during the first few months of debugging and program development. Backup diskettes made recovery relatively easy.

The remote mass storage is available via a modemtype phone communication link with the two larger computers already mentioned. Via the phone link, data are easily transmitted to the larger computer, where more extensive statistical processing and clinical report writing can be done. Peripherals on the large computer can be used to read or store information that can then be transferred to the microcomputer.

\section{Digital Input/Output Lines and Timers}

This section examines hardware timers and those devices and interfaces that channel information between the microcomputer and the external world.

Like mass storage, the $\mathrm{I} / \mathrm{O}$ devices such as the line printers on the IBM 3032 and DEC-10 are accessible via the phone lines. For the stand-alone system, a DECwriter II provides 30-cps hard copy. At the present time the DECwriter II is used to initialize the system and for program development where hard copy is desirable. A specialized CRT system discussed elsewhere in this paper is being temporarily used as a terminal for program development. Within the near future, a standard CRT terminal will be added.

Four custom-built boards handle the interfacing. A serial RS232C board, a timer board, a digital I/O board, and a buffer/latch board were built by a University electronic technician. In retrospect, purchasing previously designed and built interfacing would have considerably speeded up the laboratory development at somewhat less cost. Also, standard boards are more easily replicated for satellite systems.

The serial interface board contains four RS232C circuits, one modem circuit, and a 17-bit serial shift register. Two RS232Cs are for future expansion; one is used to communicate with the DECwriter, and one is dedicated for the CRT terminal to be ordered. The 17-bit serial shift register controls the audio device yet to be discussed. This circuitry automatically shifts out 17 bits after 2 bytes are software loaded into the designated addresses. Bit 17 is a control bit peculiar to the audio device and is automatically added by the circuitry.

The timer board contains two 8253 programmable interval timer chips (Intel Corp., 1975) capable for use as a one-shot, square-wave generator, interval timer, event counter, or software- or hardware-triggered strobe Each chip has three separate 16-bit interval timers with a clock rate capability of dc to $2 \mathrm{MHz}$. Presently, two timers have a $1-\mathrm{msec}$ time base, two a $1-\mathrm{sec}$ time base, and two are programmable from $1 \mathrm{msec}$ to $1 \mathrm{sec}$.

The interval timers simplify program control where delays are required. One timer is used for timing disk access operations on the floppy disks instead of using the software loops provided by the manufacturer. Using this timer, it is possible, for example, to transfer characters from the DEC-10 to our computer with no loss during disk access time. A second timer is used as a time-of-day clock, and the others are available for experimental control. They may, for example, be used to time subject responses, such as in reaction time experiments, or to generate timed flashes in tachistoscopic work. Since each timer can be read "on the fly" without disrupting the counting, such experimental control is precise and easy to use. The strobing capability is useful for visual evoked response experiments. The event counter mode (a divide-by- $\mathbf{N}$ counter) is useful for counting external events. It could be used, for example, to count finger tapping measures used in neuropsychological work.

When the timers are used for interval timing, an output flag is set upon terminal count. This is tied directly to an input of a Z80 parallel $\mathrm{I} / \mathrm{O}$ interface chip (PIO), which can be set to interrupt the microprocessor. In other words, an interrupt service routine can be used in conjunction with a time-out condition. This capability provides a mechanism for multitasking should the need arise.

Four Z80 PIOs are provided on the digital I/O board, 
giving a total of eight ports or $64 \mathrm{I} / 0$ lines. The PIO ports can be operated in input or output mode, or individual bits are assignable under program control as either an input or output (Osborne, 1976). Each I/O line that interacts with the external world is unidirectionally buffered to protect the PIOs from external shorts or accidental application of excessive voltage. Because of this buffering, these bits were preassigned at the time of system development. Bits assigned as input lines are also capable of generating interrupts. Software controls whether the interrupt occurs on a positive- or negativegoing pulse edge. If an interrupt is enabled for a particular bit and an interrupt occurs, the port for that input line outputs an address byte telling the microprocessor in which port the interrupt occurred and which interrupt service routine is to be executed. The program must determine which bit within the port caused the interrupt. By careful assignment of input lines, it is possible that, for any given experiment, only one interrupt is likely to occur for each port. This makes interrupt decoding unnecessary. Priorities are arbitrated through daisy chain logic.

The various $\mathrm{I} / \mathrm{O}$ lines are assigned to perform such functions as: (1) receive input from a pair of reaction time keys, (2) receive input from a numeric keyboard, (3) sense flag conditions on the HP graphics translator, and (4) accept outputs from the interval timers.

Although an HP 1311A with graphics translator is set up to function as a tachistoscope (T-scope), the laboratory also contains a three-channel Scientific Prototype T-scope with automatic stimulus changer. Each channel, as well as stimulus changer, buzzer, and fixation point, is directly controlled via optoisolators by $\mathrm{I} / \mathrm{O}$ lines, thereby greatly expanding the logic and timing functions available for T-scope work.

Finally, $11 \mathrm{I} / \mathrm{O}$ lines communicate with the HP 1350A graphics translator. This Hewlett-Packard instrument uses the IEEE 488-1975 instrumentation bus specifications. Since the $1350 \mathrm{~A}$ is the only instrument on the bus, handshaking is simplified, and software simulation of the necessary signals has been done. Motorola now has a chip, the MC68488, that in conjunction with bus driver ICs can automatically handle all handshaking needed to interface with the IEEE 488 bus.

The final interface board contains buffers, latches, and flags. All $\mathrm{I} / \mathrm{O}$ lines to the external world are buffered to protect the PIOs and the microprocessor. All input lines intended to generate interrupts have flags that are set upon interrupt and subsequently must be cleared by the service routine. The flags prevent lower priority interrupt requests from being lost in case a higher priority interrupt is being serviced.

Latches, as a method of retaining signal values, are provided for the numeric keyboard. This ensures that all inputs from this device are captured.

\section{Audio/Visual/Subject Response Keys}

We wanted to be able to present experimental in- structions in a standard format with no inflection changes or nonverbal behavioral cues. Both auditory and visual modalities were chosen. Various ways of presenting verbal material are available in the market place, including voice synthesizers, digitized speech, and tape recorders, and each has its drawbacks. The quality of speech generated from voice synthesizers is less than optimal, digitized speech requires vast amounts of memory, and tape recorders must be sequentially searched for the desired message, creating undesirable access times.

An alternative to these devices and the one in use in the laboratory is the RA-4096 random access audio unit built by Education and Information Systems, Inc. This unit was developed for use on the PLATO CAI system at the University of Illinois (Jones, 1976). It is a random access device ( $1 / 2-\mathrm{sec}$ average access time) that uses a 15 -in. mylar magnetic disk on which goodquality messages can be recorded. Messages can be played back under microcomputer control in any sequence. The verbal responses of a subject can also be recorded. The mylar disk and the record/playback head are position driven by a 15-psi air supply. Most hospitals have such a supply, but the lab uses air from a 244-cu-ft compressed air tank with a Variflo 1- to 100-psi adjustable regulator. This tank of air ensures mobility.

To address the RA-4096, a serial shift register sends two 17-bit words with track number (radial position), sector (angular position), length (number of sectors), and play or record status. There are 128 tracks and 32 sectors constituting 4,096 separately addressable locations of approximately $1 / 3 \mathrm{sec}$ each. The tracks are divided into groups of four, enabling the use of continuous messages from $1 / 3 \mathrm{sec}$ to about $43 \mathrm{sec}$ in length. Longer messages can be recorded and with proper synchronization a total of $23 \mathrm{~min}$ is available.

This device is used in the lab to deliver experimental instructions, ask questions, and give error corrections. For example, the Kelly Role Construct Repertory Test (Kelly, 1955) is automated on the system. As part of the test procedure, it asks for the names of acquaintances in the subject's present or past. Messages are prerecorded, such as "Thank you, now give the name of your mother, stepmother, or the person who is or was most like a mother to you," and then played back at the appropriate time. If a subject is asked this question and fails to answer within a specified time, a message asks the subject to respond, then repeats the original question. At other times in the Kelly rep test, the subject is asked to choose among three names. If an extraneous choice is made, the computer responds with a message indicating the particular choice was not one of the three possible choices. The question is repeated. As well as easing test administration, the audio modality is useful for subjects who cannot read.

The visual display is a companion to the audio system and is used for such experiments/tests as reaction time, T-scope work, and the Kelly rep test. The heart of the visual presentation system is a Hewlett-Packard 
(HP) 1350A graphics translator. It is a sophisticated character generator and vector graphics driver for a nonstorage-type CRT, in this instance, a HP 1311A. The 1350A has $2 \mathrm{~K}$ 32-bit words of RAM that can be constituted as 32 separately addressable and erasable files. The RAM is continually accessed to generate vectors and characters on from one to four directed-beam CRTs. Each memory word may contain either an upper- or lowercase ASCII character or a vector coordinate. All ASCIl characters are vector generated and may be displayed in four sizes $(1,2 \mathrm{x}$, $4 x, 8 x)$ and two orientations ( 0 and $90 \mathrm{deg}$ ). Vectors can be drawn between any of the 1,022 by 1,023 points.

The 1350A uses the IEEE 488 bus or an optional RS232C. Instructions to generate specific displays are received in ASCII format and include the ability to blank or unblank named files, erase files, send text, plot absolute, determine character size and orientation, as well as others. In our system a simple FORTRAN write statement, with $1350 \mathrm{~A}$ commands given in literals within the format statement, is all that is needed.

Because of the ability to selectively blank and unblank files, flashing and moving displays can be generated. By using auxilliary outputs to sense refresh scanning and/or external CRT unblanking, the 1350A with the 1311A CRT or similar scope can be used to flash displays for short duration. It takes a minimum of $6 \mathrm{msec}$ to blank one file and turn on another.

The $1311 \mathrm{~A}$ is an $8 \frac{1}{2} \times 11 \mathrm{in}$. CRT with a high writing speed $(25 \mathrm{~cm} /$ microsec) and a fast settling time (300 nsec to within one $.38-\mathrm{mm}$ spot diameter). The screen is coated with a green P31 phosphor that decays to $10 \%$ persistence in 38 microsec (Hewlett-Packard, Note 1) and to $1 \%$ persistence in 250 microsec. This fast decay time makes the scope suitable for T-scope-type work. A TTL blanking option enables high-speed screen blanking and unblanking of single displays.

The 1350A and 1311A combination has proved to meet our visual display needs with minimal overhead. The 1350A eliminates software generation of characters and enables us to generate complex displays with ease. As a simple example, the Kelly rep test presents a name, such as John; a construct/contrast pair, such as happy/sad; and a 6-point scale (1 to 6) with descriptors for each number, such as very, slightly, and the like. The subject, via the audio system, is asked to rate John on a happy/sad dimension, entering the rating on the numeric keyboard.

The 1350A and 1311 A has also proved helpful during development. Via software, the 1311A simulates an input terminal with scrolling. Information is entered on the DECwriter II and echoed on the scope, the DECwriter, or both. Time and paper are saved by adapting the visual display this way.

There are two minor deficiencies worth noting.
First, the characters generated on the 1311A consist of vectors that overlap at the ends. Where this overlap occurs, increased brightness is produced. Thus characters are uneven in brightness, which presents a problem in illumination for perceptual research. HewlettPackard claims to have solved this problem with a new z-axis board for the 1311A. Another solution might be to choose characters with similar illumination characteristics. The second problem is the acceptance of characters by the $1350 \mathrm{~A}$ from the IEEE 488 bus. If a certain type of formatting error occurs, the $1350 \mathrm{~A}$ will not return a data-accepted pulse, and thus will accept no more characters until the bus is cleared.

A binary decoded keyboard with numbers from zero to nine accepts information from a subject. When, for example, a person is asked to rate an acquaintance on a 1- to 6-point scale, the rating can be entered by hitting the corresponding key.

The audio/visual/response keyboard configuration provides a versatile method of interacting with research subjects and clinical patients. Shortly, we expect to have a CRT terminal and a means for physiological recording.

An important consideration is cost. Table 1 gives a breakdown for hardware costs, excluding the custombuilt boards. Since we are recommending the use of standard off-the-shelf interfacing, similar interfaces are listed. Because of rapid changes in the computer industry, prices may change rapidly.

If the $1350 \mathrm{~A}$ and $1311 \mathrm{~A}$ are eliminated, the cost is reduced considerably. For applications where these features are not required, a powerful system for experimental control is available at considerably less cost.

The system described here appears to have met our design prerequisites of flexibiltiy in experimental control, ease of data handling, system mobility, operation simplicity, and minimal turnaround time for clinical feedback. The research and clinical potential for such a system is enormous.

Table 1 Component Costs for Laboratory System

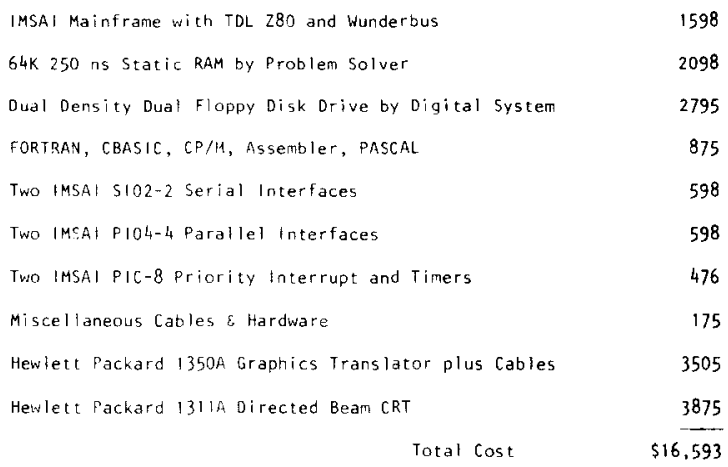




\section{REFERENCE NOTE}

1. Hewlett-Packard. Principles of cathode-ray tubes, phosphors, and high-speed oscillography. Application Note 115. Palo Alto: Author.

\section{REFERENCES}

Bowles, K. L., \& Hollan, J. D. An introduction to the UCSD PASCAL system. Behavior Research Methods \& Instrumentation, 1978, 10, 531-534.

Cromwell, R. L., \& Spaulding, W. A mictoprocessor-based psychopathology laboratory: I. Why bother? Behavior Research Methods \& Instrumentation, 1979, 11, 241-242.

Huntzinger, R. S., \& Space, L. G. A microprocessor-based psychopathology laboratory: IV. Software design. Behavior Research Methods \& Instrumentation, 1979, 11, 253-256.

INTEL CORPORATION. Intel 8080 microcomputer systems user's manual, September 1975.

JoNEs, A. Operating manual for the RA-4096 random access audio unit. Champaign, Ill: Education and Information Systems, Inc. 1976.

KELlY, G. A. The psychology of personal constructs (2 vols.). New York: Norton, 1955.

Osborne, A. An introduction to microcomputers, Volume 11: Some real products. Berkeley: Adam Osborne and Associates, Inc., 1976.

Spaulding, W. D., \& Space, L. G. A microprocessor-based psychopathology laboratory: II. Research goals and design prerequisites. Behavior Research Methods \& Instrumentation, 1979, 11, 243-246. 